Prepared for the U.S. Department of Energy

under Contract DE-AC05-76RL01830

\title{
Subsurface Biogeochemical Research FY11 Second Quarter Performance Measure
}

TD Scheibe

March 2011

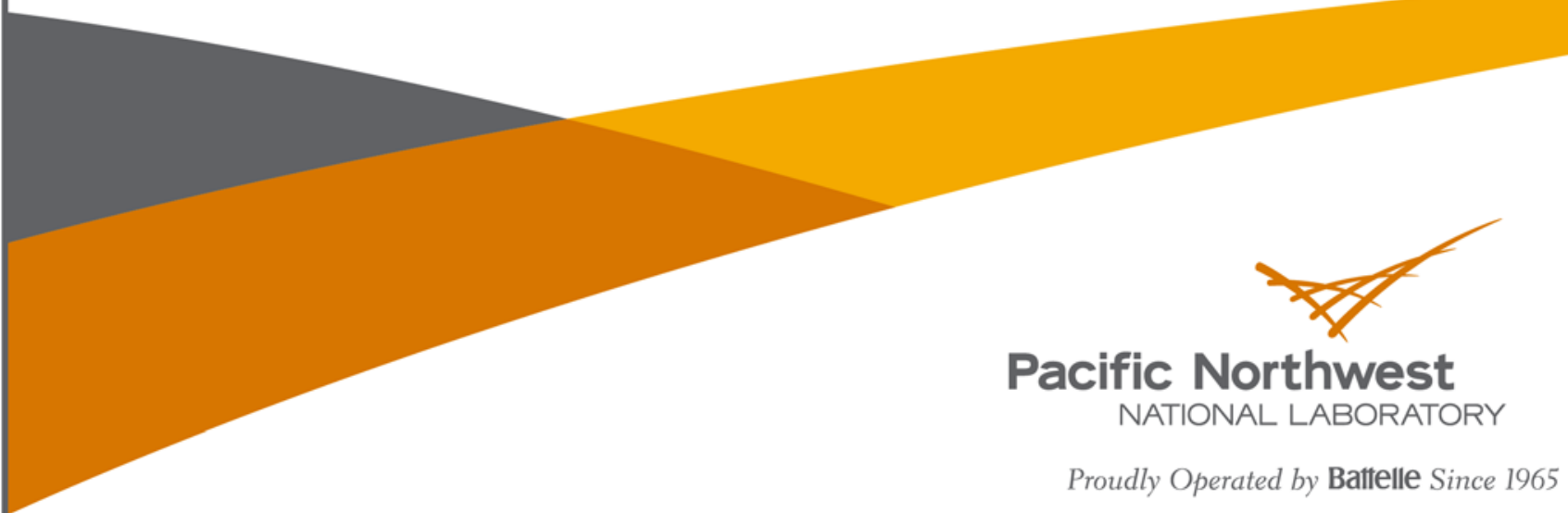






\title{
DISCLAIMER
}

This report was prepared as an account of work sponsored by an agency of the United States Government. Neither the United States Government nor any agency thereof, nor Battelle Memorial Institute, nor any of their employees, makes any warranty, express or implied, or assumes any legal liability or responsibility for the accuracy, completeness, or usefulness of any information, apparatus, product, or process disclosed, or represents that its use would not infringe privately owned rights. Reference herein to any specific commercial product, process, or service by trade name, trademark, manufacturer, or otherwise does not necessarily constitute or imply its endorsement, recommendation, or favoring by the United States Government or any agency thereof, or Battelle Memorial Institute. The views and opinions of authors expressed herein do not necessarily state or reflect those of the United States Government or any agency thereof.

\author{
PACIFIC NORTHWEST NATIONAL LABORATORY \\ operated by \\ BATTELLE \\ for the \\ UNITED STATES DEPARTMENT OF ENERGY \\ under Contract DE-AC05-76RL01830
}

Printed in the United States of America
Available to DOE and DOE contractors from the Office of Scientific and Technical Information,
P.O. Box 62, Oak Ridge, TN 37831-0062;
ph: (865) 576-8401
fax: $(865)$ 576-5728
email: reports@adonis.osti.gov

\begin{abstract}
Available to the public from the National Technical Information Service, U.S. Department of Commerce, 5285 Port Royal Rd., Springfield, VA 22161 ph: (800) 553-6847 fax: $(703) 605-6900$ email: orders@ntis.fedworld.gov online ordering: http://www.ntis.gov/ordering.htm
\end{abstract}

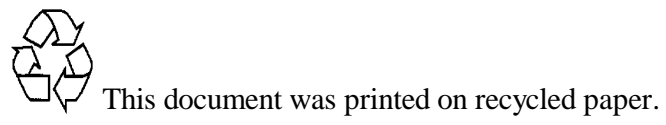




\section{Subsurface Biogeochemical Research FY11 Second Quarter Performance Measure}

TD Scheibe

March 2011

Prepared for the U.S. Department of Energy under Contract DE-AC05-76RL01830

Pacific Northwest National Laboratory

Richland, Washington 99352 


\section{US Department of Energy, Office of Science Office of Biological and Environmental Research (BER) Subsurface Biogeochemical Research (SBR) FY11 Second Quarter Performance Measure}

The SBR Long Term Measure for 2011 under the Performance Assessment Rating Tool (PART) measure is to "Refine subsurface transport models by developing computational methods to link important processes impacting contaminant transport at smaller scales to the field scale." The second quarter performance measure is to "Provide a report on computational methods linking genome-enabled understanding of microbial metabolism with reactive transport models to describe processes impacting contaminant transport in the subsurface." Microorganisms such as bacteria are by definition small (typically on the order of a micron in size), and their behavior is controlled by their local biogeochemical environment (typically within a single pore or a biofilm on a grain surface, on the order of tens of microns in size). However, their metabolic activity exerts strong influence on the transport and fate of groundwater contaminants of significant concern at DOE sites, in contaminant plumes with spatial extents of meters to kilometers. This report describes progress and key findings from research aimed at integrating models of microbial metabolism based on genomic information (small scale) with models of contaminant fate and transport in aquifers (field scale).

\section{Background}

Subsurface microbiology is a relatively new field of scientific investigation. As recently as 25 years ago, it was widely believed that life in the subsurface was limited to soils and perhaps shallow unconfined aquifers. However, during the past two decades there has been an explosion of new information about the abundance, spatial distribution, and activity of microorganisms in the subsurface environment. In 1987, Dr. Derek Lovley discovered a new microorganism, Geobacter metallireducens (Figure 1), that was the first organism found to oxidize organic compounds to carbon dioxide with iron oxides as the electron acceptor. In other words, Geobacter metallireducens gains its energy by using solid rust-like iron minerals in the same way that humans use oxygen. Geobacter metallireducens and other Geobacter species that have subsequently been discovered provide a model for important iron transformations on modern earth and may explain geological phenomena, such as the massive accumulation of magnetite in ancient iron formations. Geobacter species are also of interest because of their

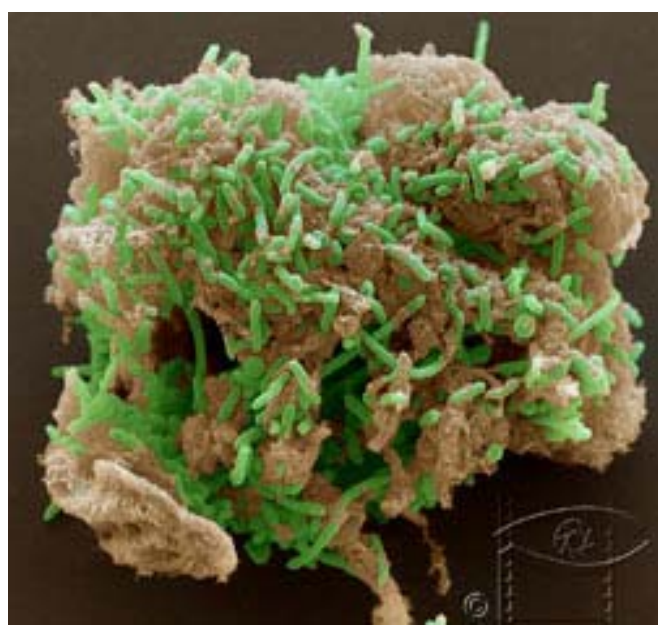

Figure 1 Geobacter metallireducens. (c) Eye of Science. role in environmental restoration. For example, Geobacter species can destroy petroleum contaminants in groundwater by oxidizing these compounds to harmless carbon dioxide. Of particular interest to the Department of Energy (DOE), Geobacter species are also able to convert radioactive metal groundwater contaminants such as uranium into an immobile mineral form, thus inhibiting its movement to environmental 
receptor points such as streams and wells. As understanding of the functioning of Geobacter species has improved it has been shown to be possible to use this information to modify environmental conditions in order to accelerate the rate of contaminant degradation or immobilization.

At a Uranium Mill Tailings Remedial Action (UMTRA) site near the town of Rifle, CO (referred to here as the Rifle Site; see Figure 2), a DOE-sponsored research team has been conducting field research on the ability of Geobacter species and other subsurface microorganisms to immobilize uranium in groundwater and keep it from migrating to the nearby Colorado River.

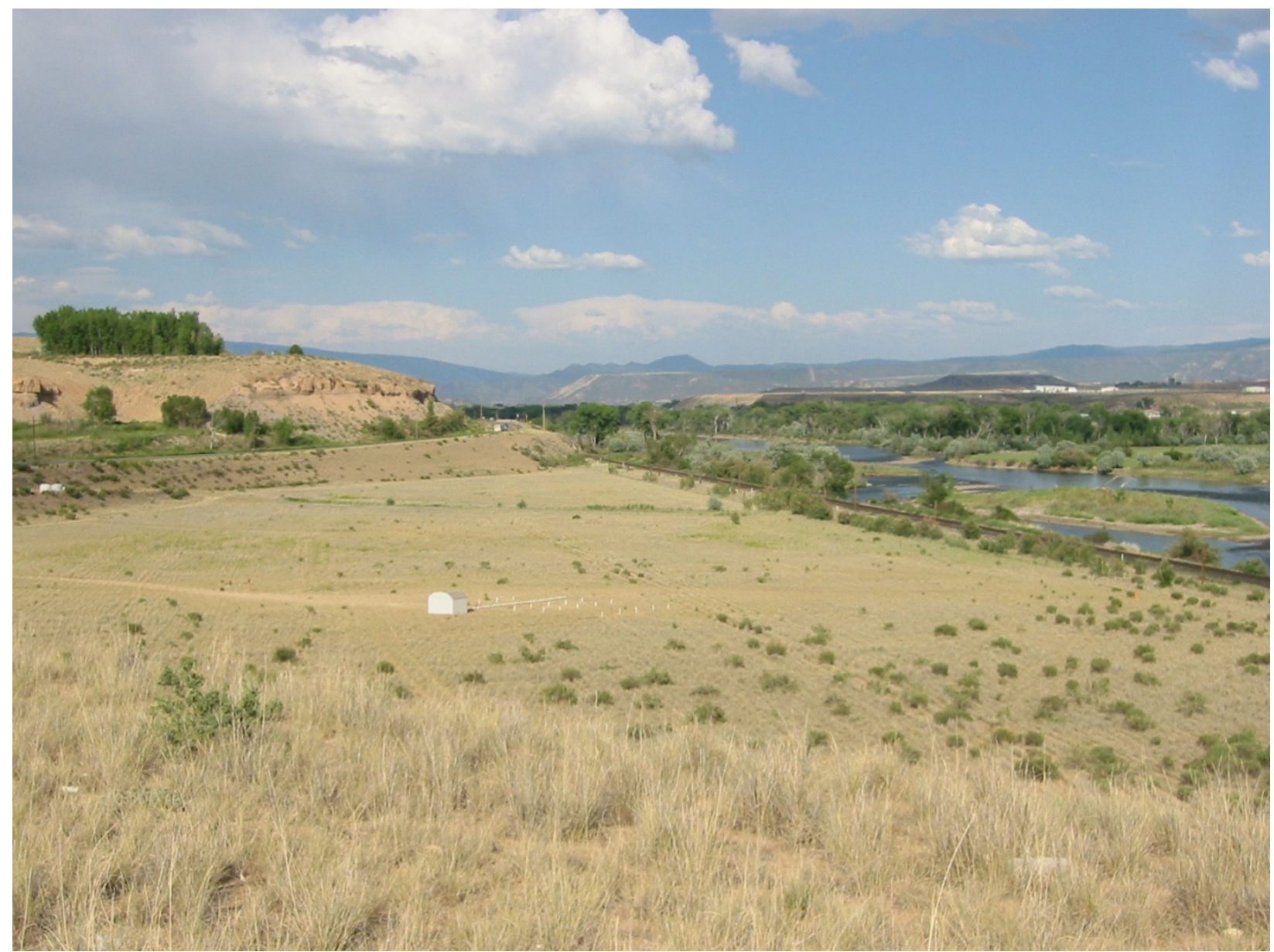

Figure 2 Photograph of the Rifle Site. The shed and wellheads in the center of the photograph delineate one of the experimental field plots at which reductive immobilization of uranium by native microorganisms is being studied. The Colorado River is in the right side of the photograph. Photo courtesy of Dr. Phil Long, PNNL.

Rifle is the site of a former uranium mine, and exposure of the mine tailings to oxygen leads to the release of several oxidized metals including uranium, which can then move with the groundwater toward the river. The Rifle research team, led by Dr. Philip Long, has performed a series of field experiments in which they amended groundwater with dilute solutions of acetate. This soluble form of organic carbon is utilized as an electron donor by microorganisms, most notably Geobacter species which have been observed to "bloom" in response to acetate addition. Geobacters use natural iron oxide minerals as their primary electron acceptor, but can also use 
uranium as an electron acceptor. This microbial reaction process leads to chemical reduction of uranium from the $\mathrm{U}(\mathrm{VI})$ valence state to the $\mathrm{U}(\mathrm{IV})$ valence state, which in turn causes a significant reduction in uranium solubility leading to precipitation of uranium minerals in the aquifer sediments. Since U(VI) is relatively soluble and moves with groundwater whereas U(IV) is relatively insoluble and immobile, this biogeochemical transformation can significantly reduce the amount of uranium transported to the river.

\section{Numerical Simulation of Microbially-Mediated Metal Reduction}

The SBR Program supports a long-term PART measure to "provide sufficient scientific understanding such that DOE sites would be able to incorporate physical, chemical and biological processes into decision making for environmental remediation and long-term stewardship." Scientifically sound decisions rely in part on our ability to quantitatively predict the future outcomes of a set of alternative management options under consideration, and define uncertainties associated with those predictions, based to the extent possible on fundamental understanding of the physical, chemical, and biological processes involved. Ideally, such simulations could also be used to design an optimal strategy for remediation and monitoring of a contaminated site. In the context of scientific research, numerical simulations can also be used to support experimental design (pre-modeling) and to integrate large and diverse data and information into a quantitative framework that supports interpretation of experimental observations and hypothesis testing (post-modeling).

A variety of quantitative models of groundwater flow, solute transport, and biogeochemical reactions have been posed and formulated into numerical computer codes. At the Rifle Site, the code HYDROGEOCHEM has been applied to Rifle Site field experiments (Yabusaki et al., 2007; Fang et al., 2009). This code can incorporate mathematical representations of a variety of processes including physical processes (e.g., water flow and advective solute transport, dispersion, aquifer recharge at surface and wells), chemical processes (e.g., aqueous complexation reactions, grain surface sorption of uranium and other chemical species, abiotic reduction/oxidation reactions) and microbial processes (e.g., microbial attachment to and detachment from grain surfaces, microbially-mediated iron, uranium, and sulfate reduction). These mathematical representations typically take the form of a set of differential equations in space and time, which are subsequently solved by numerical approximation methods. Of particular interest here are those equations describing the microbially-mediated redox reactions (terminal electron accepting processes or TEAPs). These reactions are usually slow relative to aqueous geochemical speciation reactions and relative to transport processes (advection and dispersion), so must be treated as kinetic reactions. This requires specification of rate formulations for TEAPs. Conventionally, these are treated using various forms of Monod rate laws, which depend in non-linear fashion on the concentration of key substrates such as the electron donor (e.g., dissolved organic carbon) and electron acceptor (e.g., solid phase iron oxides). For example, Yabusaki et al. (2007) use the following rate law to describe the utilization rate of acetate (injected soluble electron donor). 


$$
R_{C}^{b i o}=-\sum_{e A=1}^{N_{e A}} \chi_{e A} \mu_{m, e A}\left(\frac{C_{c}}{K_{S, C}+C_{c}}\right)\left(\frac{C_{e A}}{K_{S, e A}+C_{e A}}\right)
$$

where $N_{\mathrm{eA}}=$ number of terminal electron acceptors, $C_{\mathrm{c}}=$ acetate concentration, $C_{\mathrm{eA}}=$ terminal electron acceptor concentration, $\mu_{\mathrm{m}, \mathrm{eA}}=$ acetate oxidation rate coefficient for the terminal electron acceptor, $K_{\mathrm{s}, \mathrm{C}}=$ half-saturation coefficient for acetate, and $K_{\mathrm{s}, \mathrm{eA}}=$ half-saturation coefficient for the terminal electron acceptor. $\chi_{\mathrm{eA}}$ is an indicator variable that turns each TEAP on and off at various times in the simulation, and depends on the concentration of the next most favorable electron acceptor. This rate law is formulated as a summation, where each term in the series represents one particular TEAP. The rate of acetate utilization associated with iron reduction, for example, is one term in this series and can be written as:

$$
R_{C, F e}^{b i o}=-\chi_{F e} \mu_{m, F e}\left(\frac{C_{c}}{K_{s, C}+C_{c}}\right)\left(\frac{C_{F e}}{K_{s, F e}+C_{F e}}\right)
$$

The two parenthetical terms on the right-hand-side are the Monod terms, with the first term representing the effect of the acetate concentration and the second term the effect of the ferric iron (Fe(III)) concentration. Three constant parameters are defined: The two half-saturation coefficients in the Monod terms $\left(K_{s, C}\right.$ and $\left.K_{s, F e}\right)$ and the acceptor-specific acetate rate coefficient $\left(\mu_{m, F e}\right)$. The $\chi_{F e}$ term is a binary function $(0,1)$ of the concentration of the next most favorable electron acceptor and is also empirically specified. Yabusaki et al. (2007) describe a stepwise calibration approach in which these model parameters/functions were selected in order to match field observations from an initial field experiment.

The above approach is very sophisticated and complex, has been successfully implemented at the Rifle Site and other sites, and represents the currently accepted state-of-the-art in biogeochemically reactive transport modeling. However, the approach does have some wellknown limitations that we aim to address through new developments:

1. The representation of the microbial reactions uses a single rate law form and a single set of parameters over all conditions. However, we know that microorganisms have encoded in their genome many alternative metabolic pathways, each of which can be activated or deactivated depending on the local environment that a particular organism experiences. For example, many Geobacter species have the ability to fix inorganic nitrogen, but can also use ammonium if it is available. Since nitrogen fixation is a metabolically expensive process, the local concentration of ammonium can be expected to significantly impact the overall metabolic rate and biomass yield of microbial acetate oxidation. The potential switching between these two pathways is not represented in the conventional model approach.

2. Calibrated reaction rate parameters provide for descriptive modeling of prior field observations, but their applicability in a predictive sense (that is, to simulate behavior under conditions or at time and length scales other than those for which they were calibrated) is unclear.

3. The conventional models do not take advantage of dramatic recent advances in genomic and proteomic characterization of subsurface microorganisms. 
The genomes of several Geobacter species and other relevant subsurface microorganisms have recently been sequenced, and computer simulations (so-called "in silico models") of microbial metabolism based on genome-scale information have been developed. Our goal in this work is couple these explicit genome-scale simulations of microbial metabolic processes with standard flow and geochemical reactive transport models, thus replacing the empirical, descriptive Monod models currently in use with mechanistically-based, predictive models. This report describes the significant progress we have made to date toward this goal, and outlines ongoing and future extensions of this work.

\section{Constraint-Based Genome-Scale In Silico Models of Microbial Metabolism}

The field of environmental microbiology has recently taken a quantum leap through developments in molecular biology such as high-throughput multiplex sequencing, high-density microarrays and environmental proteomics; these technologies provide a deluge of information on the nature and function of microbial communities in natural systems. One development of particular significance to this work is the capability to construct chemical reaction networks that describe the fundamental metabolic pathways of bacteria. These reaction networks are based on annotation of genome maps (identification of specific gene sequences with known functional expression), verified and refined through laboratory experimentation (Thiele and Palsson, 2010). As depicted in Figure 3, particular portions of the genome sequence, when expressed through the RNA transcription process, lead to generation of proteins that catalyze specific reactions. Identification of the major reactions encoded in an organism's genome provides a means to characterize the set of metabolic pathways that are available to the organism (Covert et al. 2001).

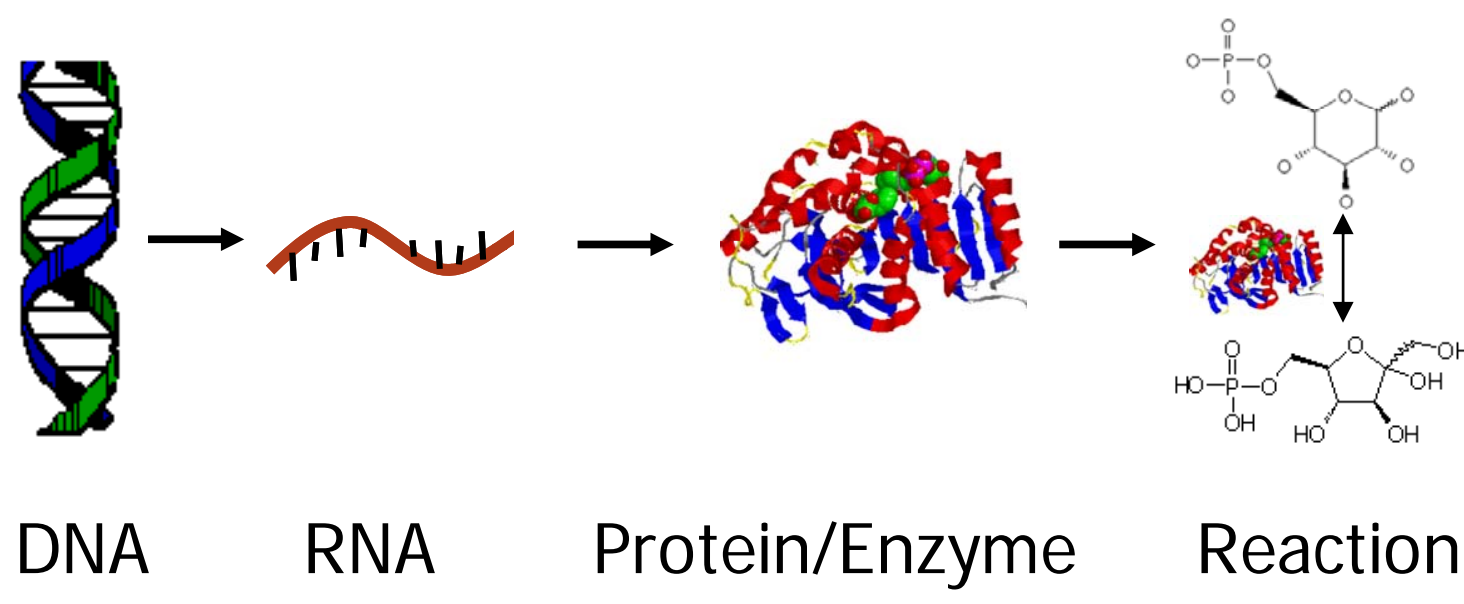

Figure 3 Schematic depiction of the process used to identify reactions in the metabolic network of an organism.

To date, full genome-scale reconstructions have been developed for over 30 organisms, and this number is expected to increase rapidly (Thiele and Palsson, 2010). Mahadevan et al. (1996) used this approach to characterize the metabolism of Geobacter sulfurreducens, an iron-reducing organism commonly found in subsurface environments. The resulting metabolic model contains over 500 reactions and 500 metabolites (reactants), including reactions central to the reduction of iron oxides coupled to acetate oxidation (a critical process at the Rifle Site). Application of this metabolic model to predict the response of $G$. sulfurreducens to specific environmental 
conditions is, however, very challenging. Each of these 500 or so reactions is kinetic in nature, with the reaction rates of each being dependent on which pathways are active and to what relative degree. Specification and parameterization of kinetic rate models for all 500 reactions under a wide range of conditions is not feasible. Other investigators have taken a kinetic network approach to simulating microbial function, but have utilized a reduced reaction network (King et al. 2009). Since one of our goals is to reduce or eliminate the use of empirical model parameters, we seek an alternative approach. One approach that allows the rates of reactions in the metabolic network to be predicted rather than prescribed is the constraint-based flux balance approach (Varma and Palsson, 1994; Price et al., 2003; Kauffman et al., 2003), which has been shown to be an effective strategy for predicting the response of organisms to environmental conditions. This approach formulates the reaction network in terms of a stoichiometric matrix $S_{i j}$, where each column of the matrix represents one reaction and each row contains the stoichiometric coefficients of a particular metabolite in each reaction. The approach is based on a steady-state flux balance, which can be applied under dynamic conditions under the assumption that shifts in microbial reaction occur quickly relative to changes in the external environment (typically a reasonable assumption in subsurface systems in which transport is controlled by slow groundwater flow and diffusion processes). We impose the requirement that at steady state, the fluxes consuming metabolites and protons must be balanced, which can be written in linear algebraic terms as:

$$
\overline{\bar{S}} \vec{v}=\overrightarrow{\mathbf{0}}
$$

Where $\vec{v}$ is a vector of fluxes of metabolites (reaction rates) through each of the $i$ reactions in the stoichiometric matrix. The solution to this equation is non-unique, but can be uniquely defined by imposing constraints on the system. In particular, the constraint-based approach applies constraints on thermodynamic directionality and enzyme capacities consistent with the organism's physiology, and additional constraints based on the maximum fluxes (uptake rates) of key metabolites through the cell membrane. The latter constraints provide a connection to the external environment of the cell, because the maximum uptake rates are a function of the external concentration. In the case of the G. sulfurreducens model, one such constraint is the uptake rate of acetate as a function of the local external concentration of acetate. Figure 4 shows experimentally measured acetate uptake rates in a chemostat with pure culture of $G$.

sulfurreducens, and indicates the existence of two distinct membrane transport mechanisms, one relatively fast and the other slower. The data in Figure 4 are fitted with Michaelis-Menten functions. These experimental functions, and similar functions for other critical extracellular metabolites such as iron oxide and ammonium, form constraints on the overall metabolic network. Solution of a linear system of equations subject to constraints is a standard optimization problem that can be solved using linear programming techniques. Optimization provides a unique solution, but requires specification of an objective function. For the case of metabolic networks, it is reasonable to assume that evolutionary pressures enforce optimal utilization of resources, which translates to an objective of maximizing growth yield for a given set of extracellular concentrations (the environment experienced by a cell). Yield is the fraction of energy gained from the metabolized substrate (acetate) that goes toward constructing cellular biomass (with the remainder of the energy going toward maintenance energy requirements). In other words, the cell is expected to allocate fluxes through its available network pathways in 
such a way so as to maximize its growth given the constraints imposed by conditions outside the cell.

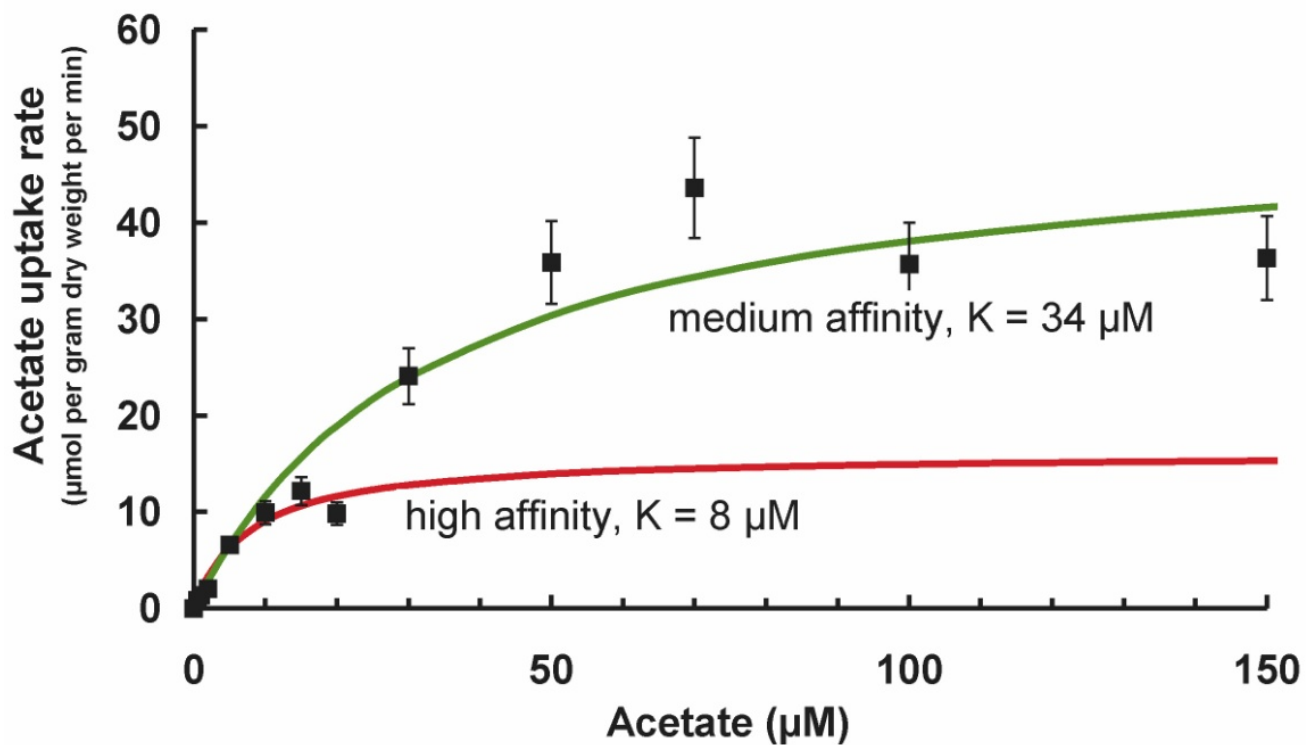

Figure 4 Measured acetate uptake rates in Geobacter sulfurreducens as a function of the extracellular concentration.

The constraint-based flux balance analysis approach has been successfully tested using a number of well-studied organisms. Useful predictions of the effects of gene deletions, preferences for particular growth substrates, and optimal growth patterns have been obtained from such models, with a success rate on the order of 70-90\% (Price et al. 2003). Genome-scale metabolic reconstruction is now a very useful element of a wide range of applications (Oberhardt et al. 2009). For example, in the manufacturing of biologically-generated products, computer simulations of microbial metabolism (so-called in silico experiments, as they are conducted on a silicon chip in a computer) can take the place of a large number of in vitro laboratory experiments that would be otherwise needed for optimal process engineering design.

\section{Coupling Genome-Scale In Silico Models of Microbial Metabolism with Subsurface Reaction Transport Simulations}

The approach used to integrate the genome-scale model of Geobacter sulfurreducens with a reactive transport model of uranium at the Rifle Site is shown schematically in Figure 5.
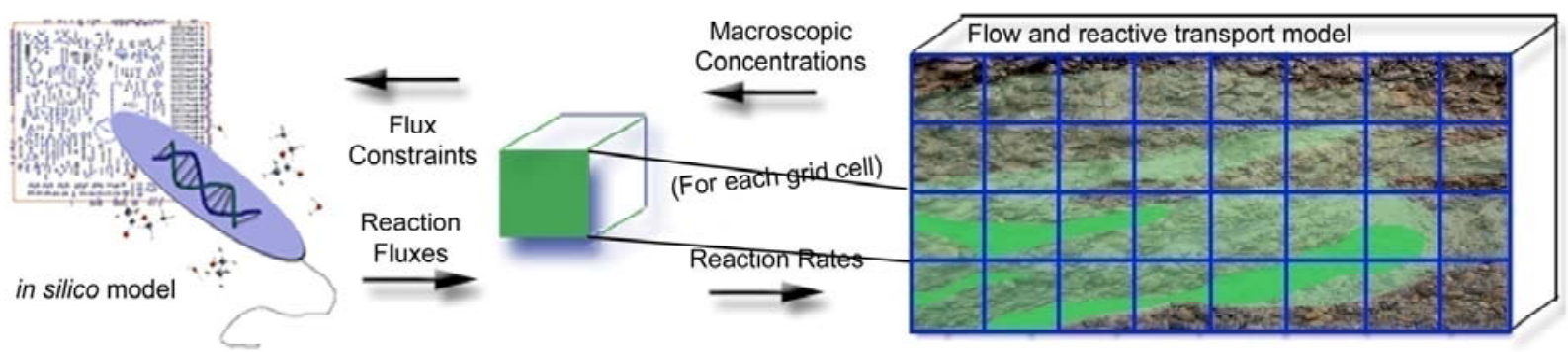

Figure 5 Schematic diagram showing the coupling of a conventional reactive transport simulator (right) with a genome-scale in silico model of microbial metabolism (left). Modified from Scheibe et al. (2009). 
Field-scale reactive transport simulators usually perform numerical approximation of partial differential equations using grid-based techniques such as finite difference, finite volume, or finite element. Typical processes represented in a subsurface reactive transport model include groundwater flow, advection (movement of solutes with flowing groundwater), dispersion (spreading of solutes associated with diffusion and unresolved velocity fluctuations), aqueous geochemical reactions between dissolved chemical species (typically treated as equilibrium), interactions of solutes with grain surfaces (sorption), and microbiological reactions (treated kinetically as discussed above). For an example at the Rifle Site, see Yabusaki et al. (2007) and Fang et al. (2009). Here, we endeavor to replace the empirically-based Monod-type kinetic rate formulations discussed above with direct simulation of microbial metabolism using a constraintbased genome scale model. To do so, we need to compute fluxes (reaction rates) through relevant reactions using the in silico model, specific to given environmental conditions that are posed in terms of concentrations of key reactants. These concentrations are known, for each grid cell, from the reactive transport simulator considering all non-microbial transport and reaction processes. For each grid cell at a given time step, these concentrations are passed to the in silico model as constraints and the in silico model is solved (a linear programming optimization problem) to determine the fluxes through the microbially-mediated reactions. These reaction fluxes are the rates that replace the Monod-based kinetic rate formulations in the conventional model, and are passed back to the reactive transport simulator to perform the microbial reaction simulation stage. This process is then repeated at each time step over the time period to be simulated.

This approach was used by our team in the first known effort to couple a constraint-based metabolic model with a reactive transport simulator, and is described in detail in Scheibe et al. (2009). We applied our approach to a one-dimensional flow and reactive transport model of uranium bioremediation at the Rifle Site, based on the original conventional model published by Yabusaki et al. (2007) that simulated an experiment performed as described in Anderson et al. (2003). The layout of injection and monitoring wells used for the subject field experiment is shown in Figure 6. The one-dimensional model essentially collapses the system behavior down to a function of distance from the injection gallery, and neglects the effects of local three-dimensional variability in physical and biogeochemical aquifer properties. Using the same description of flow and transport as used by Yabusaki et al. (2007), we replaced the Monod rate formulation for iron reduction with our in silico model of G. sulfurreducens. We considered three constraining environmental concentrations, that of 1) acetate (electron donor), 2) iron oxides (electron acceptor), and 3) ammonium (nitrogen source). In this work, we used an indirect coupling approach in which the in silico model was run for a large number of scenarios prior to execution of the reactive transport simulator. In this case, ten values of concentration of each of the three constraining chemical species were considered, giving a total of 1000 combinations that spanned the range of expected

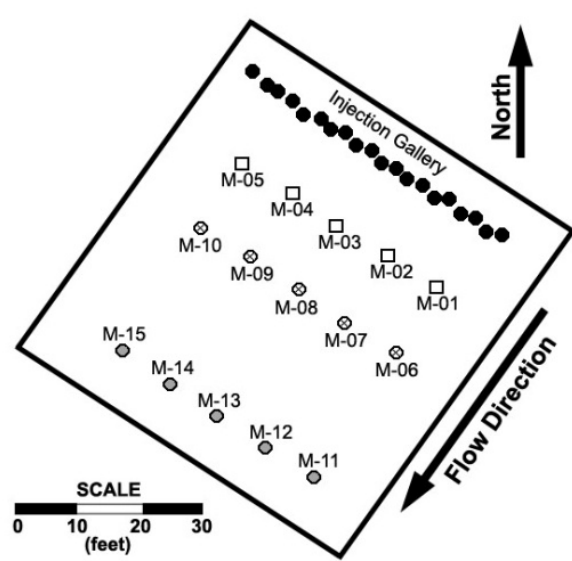

Figure 6 Layout of injection wells (solid circles) and monitoring wells (three rows, open symbols) at the Rifle Site for the experiment simulated. 
concentrations in the simulated period. For each of these combinations, the constrained flux balance was calculated and reaction fluxes were stored in a lookup table. Then, during the reactive transport simulation, instead of calling a subroutine to calculate the Monod-based reaction rates, the reactive transport code was modified to interrogate the lookup table, linearly interpolating the reaction rates based on local concentrations (at a grid cell and time step) of acetate, iron oxide, and ammonium. Use of the lookup table approach eliminates the need to call the in silico model for each grid cell and time step; since many conditions will be similar from one time step or location to another, this eliminates a significant amount of potentially redundant computation. We compared our simulation results to the results obtained by Yabusaki et al. (2007) as shown in Figure 7.
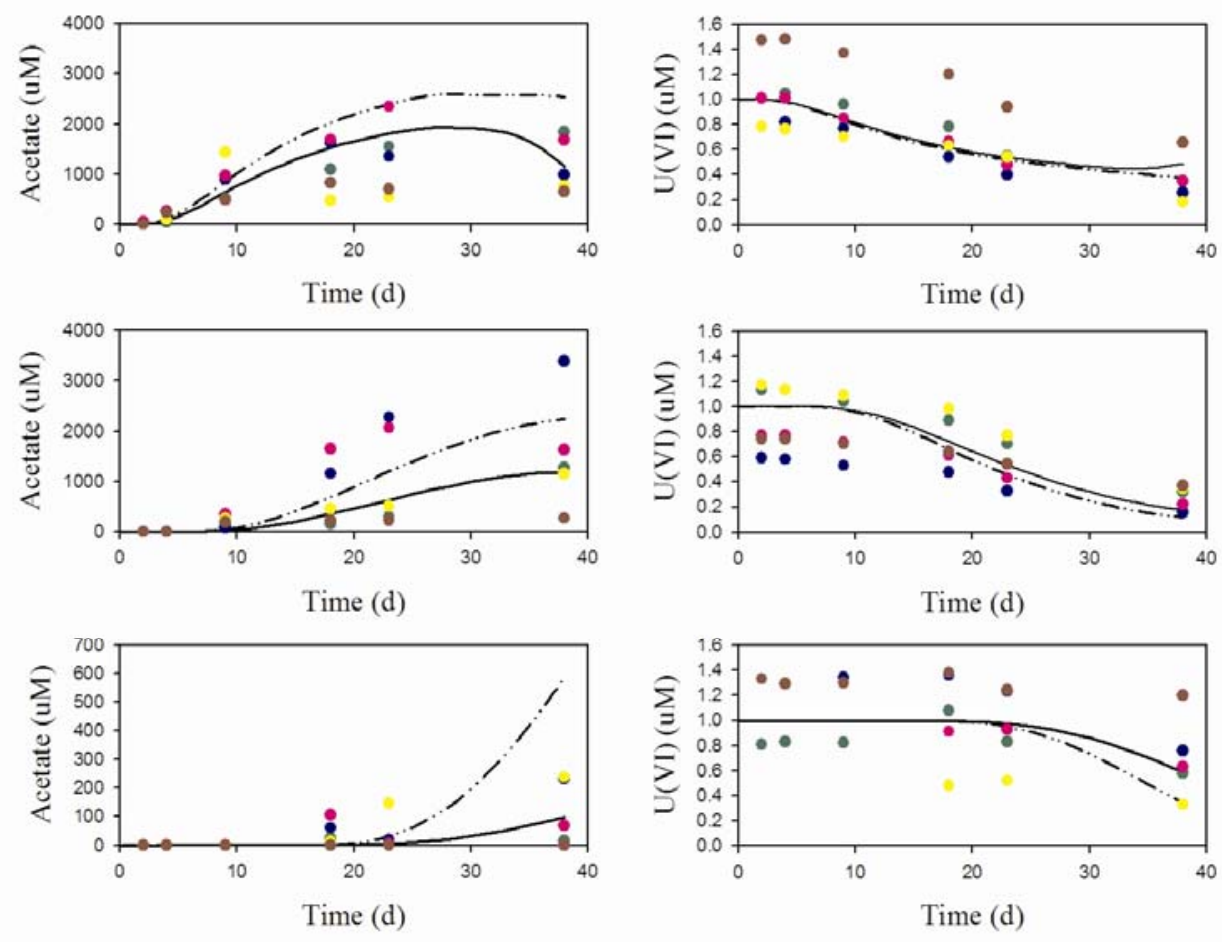

Figure 7 Comparison of simulation results obtained using the genome-scale model of G. sulfurreducens (dashed/dotted curves) with simulation results of Yabusaki et al. (2007; solid curves) and field observations from Anderson et al. (2003; colored symbols). The top plots represent the row of monitoring wells closest to the injection gallery; middle plots the second row, and bottom plots the third row)

Results are presented for two dissolved chemical species (acetate and oxidized uranium U(VI)) over the first forty days of the experiment (the iron reduction stage during which Geobacter species were dominant). As can be seen in the plots, both models reproduce the general trends of the observations in an average sense. Discrepancies between simulation results and individual data points represent the effects of three-dimensional heterogeneity that are not considered in these one-dimensional averaged models. The simulation results of Yabusaki et al. (2007), which use a conventional Monod-kinetic approach to microbial reactions, fits the observations as well as or even better than the simulations based on genome-scale modeling of microbial metabolism. 
However, the model of Yabusaki et al. (2007) requires a multi-stage calibration process with many fitted model parameters to describe the reaction rate laws. In contrast, the simulations based on the genome-scale model use a minimum of parameter modification to obtain these results. It was necessary to prescribe the initial biomass concentration, since no direct measurements were available, but the value used was within the range typically observed in shallow subsurface systems. Also, because of heterogeneity and local diffusion gradients at the pore scale, solutes are not fully mixed at the field scale as they are in the chemostat experiments on which microbial uptake rates were based. In general, because of local depletion of nutrients the concentrations in the immediate cell vicinity will be lower than those averaged overa a larger volume. Therefore, we reduced the uptake rates for acetate, iron and ammonium by one order of magnitude to generate the look-up table used for the field-scale simulation. The results of this initial model application suggest that genome-scale metabolic models can be effectively coupled with reactive transport models to describe bioremediation processes. The constraint-based metabolic modeling approach has the key advantage, compared to conventional approaches, that the growth and activity of microorganisms are predicted from detailed physiological information rather than empirically-derived rate formulations. Because of this, we expect the genome-scale modeling approach to be applicable to a wider range of environmental conditions, and to have greater predictive power, than conventional calibrated models. Further details and interpretations of this model application are provided in Scheibe et al. (2009).

Indirectly coupling reactive transport and microbial metabolism models using the lookup table approach is effective for the simple case considered above. However, in general one may wish to simulate the activity of multiple species as impacted by several chemical substrates, in which case the static lookup table approach is cumbersome. Therefore, we have also pursued a direct coupling approach in which the reactive transport simulator directly executes the constraintbased metabolic optimization for each grid node and time step. To avoid redundant computations while maintaining accuracy and generality, a constraint-based model solution pool (i.e., a dynamic lookup table) is generated during the simulation process. Each time a constraintbased solution is generated, it is stored for potential future use, indexed by the values of the constraining chemical species concentrations. When another constraint-based solution is needed, the existing pool is searched for a similar solution using an efficient tree-based search algorithm. If the set of constraining solutions is within a specified distance (tolerance) of a previouslysimulated point in the solution pool, the solution at that point is used. Otherwise, the full linear optimization of the metabolic network is performed and the new point is added to the solution pool. Details of the direct coupling approach, and its demonstration using some example problems, are presented in Fang et al. (2011).

\section{Simulating the Effects of Electron Capacitance in Geobacter on Uranium Reduction}

One intriguing aspect of Geobacter physiology that has been elucidated by genome-scale studies is the existence of reduced extracytoplasmic c-type cytochromes in planktonic cells (those not associated with a solid mineral surface) as observed by Esteve-Nunez et al. (2008). Those authors hypothesized that these cytochromes may serve as miniature capacitors, providing an extracytoplasmic sink for electron transfer and allowing Geobacter metabolism to continue during periods in which the microbes are not in contact with an Fe(III) oxide mineral surface. This is related to the observation that Geobacter species express flagella for motility to aid in 
their search for new sources of Fe(III) oxide once they have locally depleted a microsite (Childers et al. 2002), since the use of cytochromes as temporary electron capacitors may allow continuation of energy generation (metabolic activity) even when the organisms are temporarily out of contact with Fe(III) oxide surfaces (Lovley, 2008).

We have incorporated a model of electron capacitance into a reaction network describing uranium reduction mediated by Geobacter sulfurreducens (Zhao et al., 2010). This novel model divides planktonic cells into two states: Electron-loaded and electron-unloaded. A kinetic model of electron loading linked to acetate oxidation provides a rate of transfer between these two states. Simulations of an example problem based on the Rifle Site experiments were performed to evaluate the effect of including electron capacitance on the degree of uranium reduction. Incorporation of the electron loading-unloading cycle into the model led to increased rates of uranium reduction, and provides an explanation for the correlation of high rates of uranium reduction with high fractions of planktonic cells as has been observed at the Rifle Site. Global sensitivity analysis was applied to the model to determine the relative importance of a number of geochemical and microbial processes involved in Geobacter growth and uranium reduction. The results of the sensitivity analysis strongly suggests that electron capacitance in Geobacter, and the associated partitioning of microbes between planktonic and attached states, is critical to the efficiency of uranium bioremediation.

\section{Continuing Research and Extensions}

The work described above, aimed at integrating genome-scale models of microbial metabolism with reactive transport simulations of field-scale bioremediation, is groundbreaking in its utilization of new genome-scale understanding. These new approaches have the potential to transform the way in which microbially-mediated reactions are represented in numerical models of subsurface systems. Importantly, these developments are a significant step toward the implementation of a more predictive approach to reactive transport simulation that reduces reliance on empirical representations and parameter fitting. We are continuing research along these lines, focused on a number of extensions of the approach described above. This continuing research is described briefly here; results will be presented in future research publications generated by our research team and collaborators.

\section{Simulation of Community Dynamics using Genome-Scale Models}

At the Rifle Site, Geobacter species have been observed to dominate the microbial community during the iron reduction phase of bioremediation. However, other organisms and/or functional groups play a significant role in later phases. Most notably, sulfate-reducing organisms were observed to dominate after approximately forty days of acetate amendment, and this shift in community composition led to a decrease in uranium reduction rates (Anderson et al. 2003). It is clear that in most cases the simulation of a single species' metabolism such as has been presented here will not be sufficient to capture the complex behavior of subsurface microbial communities and their impact on contaminant transport. Therefore, a significant emphasis of our ongoing research is on developing multi-organism models and testing the ability of the genome-scale modeling approach to simulate community dynamics in multi-organism systems. To enhance our understanding of microbial community interactions, we have developed a new kinetic model 
for Anaeromyxobacter dehalogenans (a uranium reducer with diverse electron acceptor utilization capability) and are integrating this with the previously developed kinetic model of Desulfobacter, the genus of sulfate reducers that most actively competes with Geobacter species for added acetate at the Rifle Site. A new genome-scale constraint-based model of a related Geobacter species (Geobacter metallireducens) has recently been published (Sun et al. 2009), and models of other organisms are currently in development. A list of genome-scale metabolic network reconstructions that have been converted into predictive genome-scale models and whose predictive power has been validated against experimental data is maintained by the Systems Biology Group at the University of California San Diego (see http://gcrg.ucsd.edu/In_Silico_Organisms/Other_Organisms).

\section{Testing of Genome-Scale Models of Field-Scale Transport using Proteomic Observations}

The genome-scale model of Geobacter sulfurreducens has been applied to simulation of bioremediation at the Rifle Site as described above. This model provides predictions of which metabolic pathways are active at particular locations and times within the simulation domain. Experimental observations of the proteins expressed by microorganisms have been used to document the microbial physiology of Geobacter community members during acetate amendment at the Rifle Site (Wilkins et al. 2009). In collaboration with Rifle Site investigators, we are currently working on implementing the Geobacter sulfurreducens genome-scale model within three-dimensional reactive transport simulations of the Rifle Site and comparing the spatial and temporal distribution of simulated metabolic status with the observations of expressed proteins. This will provide an opportunity to further test the genome-scale modeling approach (preliminary results have been favorable), and will provide unique insights into the connections between spatially- and temporally-variable environmental conditions and microbial activity during bioremediation.

\section{Incorporation of Genome-Scale Models into Pore-Scale Simulations}

Microorganisms, because of their small size, respond to environmental conditions within a very localized spatial domain, probably on the order of a few tens of microns in size. However, reactive transport models typically simulate average concentrations over much larger grid blocks. In the most sophisticated models using high-performance computers, grid resolution typically is on the order of tens of centimeters or larger, and in more typical cases groundwater simulations utilize grid resolutions of tens to hundreds of meters. Successful implementation of genomescale models (which operate on the scale of individual cells) must therefore consider the effects of variability in solute concentrations at multiple length scales on effective metabolic rates. One means of exploring these scale relationships is pore-scale modeling of microbial function, in which local microenvironments can be explicitly represented. In a pore-scale model, the specific geometry of solid grains and pore spaces is explicitly characterized, and processes are resolved on a very fine grid, often as fine as a few tens of microns. We are drawing on other research by one of the co-PIs in which methods were developed and tested for pore-scale simulation of biomass dynamics (Tartakovsky et al., 2009). In that work, standard Monod kinetic formulations were used to describe rates of microbial substrate utilization and growth. We are now extending that model to incorporate the genome-scale model of iron reduction coupled to acetate oxidation 
by Geobacter sulfurreducens, and using the pore-scale model to test effects of pore-scale variability on larger-scale apparent rates.

\section{Acknowledgments}

This document was prepared by Dr. Timothy Scheibe of the Pacific Northwest National Laboratory (PNNL). It has been approved for public release as report PNNL-20292. The Pacific Northwest National Laboratory is managed by Battelle Memorial Institute under contract DEAC05-76RL01830. The research described here was conducted under the projects "Coupling In Silico Microbial Models with Reactive Transport Models to Predict the Fate of Contaminants in the Subsurface" (FY 2008-2010, Environmental Remediation Sciences Program) and "Coupled In Silico Microbial and Geochemical Reactive Transport Models: Extension to Multi-Organism Communities, Upscaling and Experimental Validation" (FY2011-2013, Subsurface Biogeochemistry Research program), both funded by the U. S. Department of Energy, Office of Science, Office of Biological and Environmental Research.

The Principal Investigator (PI) of both projects is Dr. Derek Lovley of the University of Massachusetts. Co-PIs are Dr. Radhakrishnan Mahadevan (University of Toronto) and Dr. Timothy Scheibe and Dr. Philip Long (PNNL). Several other investigators at the three participating institutions have contributed significantly to this research effort. Dr. Yilin Fang (PNNL) and Dr. Jiao Zhao (University of Toronto) have been particularly instrumental in the numerical modeling portions of this research reported here.

\section{References Cited}

Anderson, R. T., H. A. Vrionis, I. Ortiz-Bernad, C. T. Resch, P. E. Long, R. Dayvault, K. Karp, S. Marutzky, D. R. Metzler, A. Peacock, D. C. White, M. Lowe, and D. R. Lovley, "Stimulating the in situ activity of Geobacter species to remove uranium from the groundwater of a uranium-contaminated aquifer," Applied and Environmental Microbiology 69(10):5884-5891, 2003.

Childers, S. E., S. Ciufo and D. R. Lovley, "Geobacter metallireducens accesses insoluble Fe(III) oxide by chemotaxis," Nature 416(6882):767-769, 2002.

Covert, M. W., C. H. Shilling and B. Palsson, "Regulation of gene expression in flux balance models of metabolism," Journal of Theoretical Biology 213(1):73-99, 2001.

Esteve-Nunez, A., J. Sosnik, P. Visconti and D. R. Lovley, "Fluorescent properties of c-type cytochromes reveal their potential role as an extracytoplasmic electron sink in Geobacter sulfurreducens," Environmental Microbiology 10(2):497-505, 2008.

Fang, Y. L., S. B. Yabusaki, S. J. Morrison, J. P. Amonette, and P. E. Long, "Multicomponent reactive transport modeling of uranium bioremediation field experiments," Geochimica et Cosmochimica Acta 73(20):6029-6051, 2009.

Fang, Y., T. D. Scheibe, R. Mahadevan, S. Garg, P. E. Long, and D. R. Lovley, "Direct coupling of a genome-scale microbial in silico model and a groundwater reactive transport model," Journal of Contaminant Hydrology 122(1-4):96-103, 2011. 
Kauffman, K. J., P. Prakash and J. S. Edwards, "Advances in flux balance analysis," Current Opinion in Biotechnology 14(5):491-496, 2003.

King, E. L., K. Tuncay, P. Ortoleva and C. Meile, "In silico Geobacter sulfurreducens metabolism and its representation in reactive transport models," Applied and Environmental Microbiology 75(1):83-92, 2009.

Lovley, D. R., "Extracellular electron transfer: Wires, capacitors, iron lungs, and more," Geobiology 6(3):225-231, 2008.

Mahadevan, R., D. R. Bond, J. E. Butler, A. Esteve-Nunez, M. V. Coppi, B. O. Palsson, C. H. Schilling, and D. R. Lovley, "Characterization of metabolism in the Fe(III)-reducing organism Geobacter sulfurreducens by constraint-based modeling," Applied and Environmental Microbiology, 72(2):1558-1568, 2006.

Oberhardt, M. A., B. O. Palsson and J. A. Papin, "Applications of genome-scale metabolic reconstructions," Molecular Systems Biology 5:320, 2009.

Price, N. D., J. A. Papin, C. H. Schilling and B. Palsson, "Genome scale microbial in silico models: The constraints-based approach," Trends in Biotechnology 21(4):162-169, 2003.

Scheibe, T. D., R. Mahadevan, Y. Fang, S. Garg, P. E. Long and D. R. Lovley, "Coupling a Genome-Scale Metabolic Model with a Reactive Transport Model to Describe In Situ Uranium Bioremediation," Microbial Biotechnology 2(2): 274-286, 2009.

Sun, J., B. Sayyar, J. E. Butler, P. Pharkya, T. R. Fahland, I. Famili, C. H. Schilling, D. R. Lovley and R. Mahadevan, "Genome-scale constraint-based modeling of Geobacter metallireducens," BMC Systems Biology 3:15, 2009.

Tartakovsky, A. M., T. D. Scheibe, and P. Meakin, "Pore-scale model for reactive transport and biomass growth," Journal of Porous Media 12(5):417-434, 2009.

Thiele, I. and B. O. Palsson, "A protocol for generating a high-quality genome-scale metabolic reconstruction," Nature Protocols 5(1):93-121, 2010.

Varma, A. and B. Palsson, "Metabolic flux balancing: Basic concepts, scientific and practical use," Bio-Technology 12(10):994-998, 1994.

Wilkins, M. J., N. C. VerBerkmoes, K. H. Williams, S. J. Callister, P. J. Mouser, H. Elifantz, A. L. N'Guessan, B. C. Thomas, C. D. Nicora, M. B. Shah, P. Abraham, M. S. Lipton, D. R. Lovley, R. L. Hettich, P. E. Long, and J. F. Banfield, "Protogenomic monitoring of Geobacter physiology during stimulated uranium bioremediation," Applied and Environmental Microbiology 75(20):6591-6599, 2009.

Yabusaki, S. B., Y. Fang, P. E. Long, C. T. Resch, A. D. Peacock, J. Komlos, P. R. Jaffe, S. J. Morrison, R. D. Dayvault, D. C. White, and R. T. Anderson, "Uranium removal from groundwater via in situ biostimulation: Field-scale modeling of transport and biological processes," Journal of Contaminant Hydrology 93(1-4):216-235, 2007.

Zhao, J., Y. Fang, T. D. Scheibe, D. R. Lovley and R. Mahadevan, "Modeling and sensitivity analysis of electron capacitance for Geobacter in sedimentary environments," Journal of Contaminant Hydrology 112(1-4):30-44, 2010. 